\title{
Simon le mage : de la réalité économique au fantasme esthétique
}

Mario ADOBATI, Université de la Sorbonne Nouvelle (Paris 3)

Que fait-il ici, et même où se trouve cet «ici », quelle ville, quel pays, quel coin du monde maudit des dieux ? Karinthy Ferenc, Épépé (1970)

\section{Introduction}

La situation de Budaï, le narrateur d'Epépé, Hongrois débarqué par erreur dans un pays dont il est incapable de reconnaître la langue, n'est pas si différente de celle de Simon, Hongrois débarqué en France sans parler un mot de français. En opérant un renversement métaphorique, elle n'est pas non plus si éloignée de celle d'Enyedi Ildiko, Hongroise essayant de faire un film en son propre pays, dans un contexte post-communiste.

\section{De la réalité économique...}

Pour bien comprendre les conditions de création de son Simon le mage (Simon Mágus, 1999), il nous faut revenir bien longtemps en arrière, avant même la chute officielle du communisme, et retrouver les années quatre-vingts en Hongrie. La tutelle soviétique, sous la direction de Kádár, n'est pas la même que celle du voisin roumain sous Ceauşescu, et tout particulièrement dans l'industrie cinématographique, qui reflète très bien le relâchement $\begin{array}{lllll}\text { progressif } & \mathrm{du} & \text { régime } & & \text { En }\end{array}$ l'État, fraîchement entré au FMI, est détenteur d'une dette d'environ 7,5 milliards de dollars (Jeancolas 212). La Hongrie se voit contrainte d'entrer dans l'économie de marché et le secteur cinématographique est l'un des premiers visés. En 1985, puis 1987, deux lois introduisent coup sur coup les changements que la rupture politique de 1989 et le passage au capitalisme qui en découle approfondiront, par l'incitation à la privatisation des studios nationaux et l'ouverture de la production aux capitaux privés. Il s'agit de bien se rendre compte que, dans le secteur de l'industrie cinématographique en Hongrie, 1989 n'opère pas une rupture brute, mais officialise presque avec douceur la transformation déjà bien entamée d'une économie (237).

A l'inverse, l'avènement du capitalisme dans la période dite du post-communisme n'est pas la libération qu'on eût été en droit d'attendre. L'État crée, en 1991, la MMA (Magyar Mozgokép Alapítvány², qui deviendra en 1998 la MMK pour Közalapítvány), chargée de distribuer ses subventions directement aux projets de films à travers l'œuvre de différents comités. Seulement voilà : la subvention annuelle, déjà peu élevée, reste fixe durant 
les premières années de cette nouvelle décennie, malgré l'inflation croissante d'un pays en crise économique et en restructuration. Un nouveau projet de loi visant à remédier à cette situation se fait attendre, sans cesse repoussé jusqu'à son abandon à la fin des années 1990. Les conséquences sont immédiates : la production annuelle de films hongrois qui atteignait la moyenne d'une vingtaine, chute jusqu'à atteindre le seuil critique de douze et treize nouveaux longs-métrages en 1997 et 1998, ce qui faisait dire à Stéphane Bouquet : «comment va le cinéma hongrois ? Plutôt mal que bien » (31). Il faudra attendre l'année suivante pour que, péniblement, l'industrie commence à remonter la pente.

En 1998, l'année de production de Simon Mágus, c'est en moyenne 20 à 25\% d'un film que l'État parvient à subventionner. Les structures annexes comme les chaînes de télévision ou les sociétés de production privées en Hongrie ne parviennent pas à combler l'écart, puisque les premières restent méfiantes à l'idée de s'engager dans ce qui est définitivement un pari risqué (au regard, par exemple, de l'investissement dans la distribution des films américains fraîchement arrivés sur le territoire) et qu'aucune des secondes n'a les épaules assez solides pour entreprendre, seule, la production d'un unique long-métrage.

Il apparaît à présent clairement que dans les années 1990, il ne reste aux cinéastes guère d'autre choix que de se tourner vers des aides extérieures. Trois aides occidentales publiques se détachent : le Fonds ECO du CNC, le Fonds Eurimages du Conseil de l'Europe et, dans une moindre mesure, le Plan MEDIA 1 de la Communauté Européenne. D'un autre côté, les cinéastes accordent de plus en plus leur confiance aux producteurs occidentaux qui parviennent à changer leur image en engageant très en amont de la création cinématographique un travail basé sur un regain de complicité au sein de la coopération (Dekiss 61).

Il est important de saisir que dorénavant, face à l'impossibilité croissante d'envisager la production d'un film hongrois à l'aide des seuls fonds hongrois, ces cinéastes n'ont plus d'autre choix que d'intégrer une véritable culture de la coproduction. Ils se trouvent donc dans une situation délicate : encore habitués au système communiste de production, où l'État est vu comme un Père bienveillant (quoiqu'autoritaire) et dispensateur de largesses budgétaires sans attendre une quelconque rentabilité en retour (l'image n'est pas si éloignée de la vérité ${ }^{3}$ ), ils se trouvent parachutés dans un monde où le Père n'est plus la figure omnipotente des années d'enfance et où il faut apprendre à compter sur soi-même et sur les autres à travers la mise en place de coproductions plus ou moins hasardeuses qui gagnent à présent à être rentables, si leurs producteurs souhaitent continuer à obtenir des fonds par la suite. 
Enyedi appartient à une génération de cinéastes talentueux qui ont fait leurs premiers films au début des années $1990^{4}$ et qui - à l'instar de Budaï trouvant une solution à ses problèmes de communication - ont pu s'approprier cette culture de la coproduction qui a mis à la porte plus d'un géant du cinéma hongrois ${ }^{5}$. Elle appartient aussi à une génération qui a su faire sa place au milieu des anciens géants restant, ces derniers ayant une tendance très marquée à occuper l'ordre des possibles dans le secteur cinématographique, puisqu'ils dirigeaient les studios de production et siégeaient aux comités de sélection des scénarios, s'auto-privilégiant gracieusement les uns les autres ${ }^{6}$. Pour faire cette place, cette génération a profité de l'éviction de certains anciens suite aux changements économiques, mais elle s'est aussi associée, pour présenter un visage fort, à travers la création du FiFi (Fiatal Magyar Filmkészítők Társasága), l'Association des Jeunes Cinéastes Hongrois, en 1990. Enfin, cette génération, élevée sous le communisme, a fait ses armes en tant que professionnels du cinéma dans une Hongrie s'ouvrant sur l'Occident. Les artistes et techniciens partant pour l'étranger n'étaient désormais plus des émigrants ou des exilés, ils avaient la possibilité de circuler librement tout en gardant leurs attaches nationales.

La réalisatrice de Mon XX siècle (Az én XX. Századom, 1989) obtient pour son premier long-métrage la Caméra d'or au festival de Cannes de 1989 ainsi qu'un certain savoir-faire en matière de coproduction. Ce premier film est une coproduction «avec deux maisons autrichienne et ouest-allemande, et l'ICAIC cubain, avec comme protagoniste le plus célèbre acteur soviétique, le tarkovskien Oleg Jankovski» (Codelli, «Siècle, 80). Son deuxième film, Le chasseur magique (Büvös vadász, 1994), est déjà une coproduction avec la Suisse et la France. Tamas és Juli (1997) rassemble encore la France et la Hongrie, association qu'elle reproduira pour Simon Mágus, à plus grande échelle puisque pas moins de sept compagnies différentes seront appelées à participer à cette production, dégradation de la situation économique oblige. Si à partir de 1999, l'industrie hongroise semble remonter la pente, la production de Simon Mágus se situe précisément dans le creux de la vague, juste avant le début de ce nouveau départ ou, plus simplement, juste avant le moment charnière que sont la toute fin des années 1990 et le début des années 2000, le tournant du siècle, où la Hongrie sort enfin du post-communisme.

Mais pour lors, Enyedi, Hongroise, se fixe de plus en plus en France pour produire ses œuvres. Face à la difficulté de produire un film en Hongrie, nous pouvons, sur la base de Simon Mágus, explorer deux hypothèses de recherche attachées à un phénomène d'expatriation. La première, explorée au cours des paragraphes précédents et ancrée dans les champs de l'histoire et de l'économie du cinéma, veut que cette situation ait pour 
conséquence l'expatriation économique de la réalisatrice. La seconde - et qui me semble aussi la plus prolifique -, ancrée dans les champs de l'esthétique et de la poétique du film, veut qu'Enyedi intègre à son œuvre un ensemble d'éléments disparates construisant un discours relatif aux difficultés de production en Hongrie.

Cette transfusion de problématiques économiques vers des problématiques esthétiques pose peut-être avant tout une question d'ordre herméneutique, puisque malgré l'analyse qui suivra, il n'y a aucune certitude qu'Enyedi ait réellement pensé son film ainsi. Au terrible reproche de la surinterprétation, deux éléments de réponse me semblent porteurs d'une certaine vérité épistémologique. Commençons par nous rappeler un article de Pierre Boulez, «Stravinsky demeure », dans lequel il proposait une analyse du Sacre du printemps tout à fait révolutionnaire et qui ne pouvait venir que du père du sérialisme intégral, puisqu'elle s'attachait à mettre en lumière tous les jeux de structures qu'il y trouvait. Il fut accusé d'avoir trouvé des structures auxquelles Stravinsky n'avait pas pensé, ce à quoi il répondit :

Dois-je répéter que je n'ai pas prétendu découvrir un processus créateur, mais me rendre compte du résultat, les rapports arithmétiques étant les seuls tangibles ? Si j'ai pu remarquer toutes ces caractéristiques structurelles, c'est qu'elles s'y trouvent, et peu m'importe alors si elles ont été mises en œuvre consciemment ou inconsciemment, et avec quel degré d'acuité dans l'intelligence de la conception, ou encore avec quelles interférences entre le travail et le «génie ». (142)

Il importe d'analyser l'œuvre telle qu'elle est, en dehors de toute considération poïétique ou génétique $^{7}$. D'autre part, et plus simplement, «il est loin d'être garanti que l'auteur possède toutes les clefs de sa propre création »(Lengyel 266).

\section{... Au fantasme esthétique}

Nous ouvrons ainsi des territoires nouveaux à l'analyse, qui nous permettent d'énoncer l'hypothèse selon laquelle Enyedi recrée symboliquement les difficultés économiques liées à un système de production cinématographique en restructuration dans son film, afin de mieux s'en échapper. Nous allons voir à présent qu'Enyedi prend le contre-pied du réalisme magique, en opposant deux formes de magie associées l'une à l'économie, l'autre à l'humain, afin de proposer la critique d'un système de production impossible, reflet de l'enfoncement forcé du capitalisme dans un système économique préexistant (ce qui forme la période post-communiste). Son expatriation à Paris est l'occasion de renforcer son discours par l'inscription de ces problématiques dans un cadre symbolique fort. Ici se justifie la remarque de John Cunningham qui avançait que «la nouvelle ère semble aussi avoir 
encouragé un tournant vers le mysticisme et le fantastique » (146) : le magique est propice au symbolisme et à la métaphore, et en se re-territorialisant dans le réalisme, il permet d'en critiquer les aspérités.

Pour ce faire, Simon, mage utilisant ses pouvoirs pour la résolution d'affaires criminelles, arrive à Paris, depuis Budapest, par la Gare de l'Est. Appelé suite à un meurtre, il s’y rend immédiatement. Mais deux rencontres le retiennent à Paris : Jeanne, jeune française dont il tombe éperdument amoureux, et Péter ${ }^{8}$, son vieil ennemi, mage assoiffé de célébrité et d'argent. Il finit par accepter le défi de ce dernier : s'enterrer pendant trois jours et revenir. Mais sa promesse à Jeanne de la retrouver le lie...

Il y a donc, dans le film d'Enyedi, deux magies différentes qui sont à l'œuvre. La première est la magie des mages : Simon la connaît ; Péter ne connaît qu'elle. Des deux magies, elle est paradoxalement celle qui se remarque le plus et celle qui est le moins montrée. De plus, sa mise en scène s'accompagne toujours d'éléments à résonance négative, et pour cause, elle est porteuse de la première partie du message que l'on peut retrouver dans l'œuvre de la réalisatrice.

Lors de la séquence chez le préfet, où Simon (joué par le célèbre acteur hongrois Péter Andorai) négocie son paiement pour la résolution du meurtre à venir, Enyedi nous montre en Simon le mage. Personnage cynique, désabusé, matérialiste, il est ici pour vendre sa magie, ce qu'il fait le plus bassement possible, en demandant à être payé d'avance, en espèces, puis en recomptant ostensiblement la somme, s'amusant de la gène très théâtralisée des personnes présentes. Dès le départ, Enyedi lie la magie à l'argent et à la cupidité qui en découle.

La résolution du meurtre se déroule selon des modalités similaires, où le maître mot est désinvolture : arrivant sur les lieux du crime, il demande à être laissé seul, s'installe puis s'endort, contre toute attente éthique. C'est que la résolution du meurtre ne passe pas par sa magie, ce sont les plantes qu'il désigne comme témoins deux fois silencieuses du drame. En définitive, ce n'est pas lui qui résout le meurtre, mais un oscilloscope, un voltmètre et un biologiste, ce qu'il explique au policier qui lui demande sa méthode : «Il n’y a pas de méthode. Il n'y a pas de magie. » Nous verrons que ce n'est qu'à moitié vrai.

Le duel avec Péter, ses modalités d'acceptation, abondent encore en ce sens, puisque Simon, faussement humble, demande à Peter de lui enseigner quelque chose. Ce dernier, plein d'orgueil, lui demande s'il s'agit de lévitation ou de télékinésie, à quoi il s'entend répondre «quelques mots de français ». C'en est trop, et le duel est lancé. Et accepté, avec un détachement royal ; «pourquoi pas ?»

Mais le «pourquoi » que se pose le spectateur, c'est «pourquoi cette vision négative 
de la magie ? »C'est que la magie est un symbole, qu'il est possible de déchiffrer grâce à plusieurs clefs. Péter, ironiquement, nous en donne une : il est l'antithèse de Simon, qui fuit la gloire pénible que lui a apporté sa résolution du meurtre, puisque Péter ne vit que pour son don et pour ce qu'il lui apporte : argent, belle voiture et célébrité. «C'est une Berline. Deux cent Rolls-Royce, une Berline. », se vante-t-il fièrement lors de sa première rencontre avec son vieil ennemi.

Nous voyons poindre ici une association d'idées que nous pouvons adopter comme hypothèse de travail : la magie se présente comme l'incarnation d'un archétype occidental : le rêve américain, le capitalisme. D'autres éléments viennent confirmer cette association. L'utilisation de la musique, puisque Másik János réserve ses compositions électroniques aux séquences de sortilèges. Et lorsqu'après quelques séquences de ce type, un homme demande dans une cage d'ascenseur à un Simon lassé de sa célébrité malvenue de l'aider, «juste un signe, quelque chose, la vie est si difficile vous savez », et que Simon lui appose le pouce sur le front, sans l'intervention dans la séquence d'une quelconque musique, nous comprenons que le geste a une signification psychosomatique: un trope s'est créé, une association musique électronique-magie des Mages. L'autre magie, nous le verrons, est toujours accompagnée de musique orchestrale, mais avançons dès à présent que la musique électronique, en tant que produit d'une machine et non d'un instrument, est porteuse d'une valeur non naturelle (au sens littéral), d'un caractère déshumanisé. C'est que la magie des Mages n'est celle de l'homme que dans les contes. Ici elle n'est, elle-même, jamais qu'une machine, et certainement la plus inhumaine.

Mais revenons à la relation entre la magie et le capitalisme. L'association, certes discrète, est toutefois renforcée par un élément de taille, si l'on ose dire, puisqu'il s'agit de la grande arche de la Défense. Pourquoi transposer le défi de Simon (qui est maintenant le défi de Péter, puisqu'Enyedi opère un renversement par rapport à la légende originale), dans l'ouest de Paris ? C'est que La Défense est un symbole de puissance économique. Transposer le duel final dans ce lieu ne peut signifier qu'un lien entre la magie et le système économique occidental.

Mais qu'est donc venue chercher la réalisatrice à Paris ? Il eût été plus logique et plus fort, pour attaquer frontalement le capitalisme, de coproduire directement avec les États-Unis et de tourner sur cet autre continent. Vraisemblablement, Paris offre autre chose, la seconde magie du film: Paris, capitale de l'amour. Ce n'est plus le Paris de La Défense qui nous intéresse, et ce n'est plus la vulgaire magie des Mages. C'est le Paris romantique et la magie des amoureux, la magie humaine. Enyedi propose subtilement de la mettre en scène à travers 
deux relations mises en miroirs, qui sont celle de Simon avec Jeanne, et celle de la réalisatrice avec Paris.

Rappelons-nous de la résolution du meurtre par Simon : en vérité, ni le biologiste ni la magie n'ont résolu l'énigme. Ce sont les plantes. Les plantes, que Simon, tiré brusquement de son sommeil, voit à contre-jour, et sur lesquelles s'attarde langoureusement la caméra du chef-opérateur sur fond de musique instrumentale, avec des cordes lisses et tendues. Simon voit que les plantes ont été bien traitées, il demande au biologiste de les connecter à un ordinateur afin d'avoir le signal de leurs émotions, et de faire défiler devant celles-ci toutes les personnes ayant connu la victime. Son assassin ne manquera pas de faire réagir violemment celles qui tenaient à lui.

La magie de l'amour des plantes pour leur maitre, donc. Soulignée par une musique instrumentale, qui se déclinera sur plusieurs thèmes empruntés à Bartók Béla, à chaque apparition d'un sentiment amoureux, que ce soit entre des protagonistes ou vis-à-vis de la capitale. Nous avons bien deux leitmotiv, l'un de la magie, l'autre de l'amour, qui ne sont pas centrés sur un motif musical mais sur un type d'instrumentation. Il est intéressant de noter que cette opposition a été pensée d'entrée de jeu par la réalisatrice, qui souhaitait opposer des compositions originales et instrumentales à de la musique électronique créée pour les besoins du film. Brian Eno, pressenti pour ces compositions, a créé une bande-son qui a dû être remplacée au dernier moment par les compositions de Másik János, l'argent manquant pour payer le compositeur américain ${ }^{9}$.

Mais revenons au prologue, qui «résume le talent visionnaire d'Ildiko Enyedi » (Codelli, «Notes », 198). Le film s'ouvre sur un plan rapproché du visage de Simon au téléphone, fumant anxieusement une cigarette. On comprend en quelques mots qu'on négocie ses services pour élucider un meurtre. Ses dernières paroles sont : «Nem repulö. Vonat. Vonattal megyek ». Il décline sèchement la proposition de venir en avion : Pas d'avion. Le train. Je viens en train. Coupure, ouverture : travelling avant rapide, mais hésitant à droite et à gauche, en plongée légère sur les rails du train. Tons anormalement bleutés, ambiance étrange et mystérieuse, soutenue par des instruments à cordes et l'apparition discrète de cuivres $^{10}$ dans une écriture horizontale et caressante. Cette arrivée est composée d'un montage alterné entre cette vue presque subjective et un effet de distanciation obtenu par l'insertion de plans sur le train circulant.

Pour le spectateur, cette arrivée en Gare de l'Est est vraiment vécue comme une échappée hors du temps. L'atemporalisation de cette séquence, créée par la combinaison du travail du compositeur et du chef-opérateur, contribue à une sensation d'éveil à la vie. Cet 
éveil, c'est naturellement une façon pour la réalisatrice de prendre le spectateur par la main pour le faire entrer dans la ville, dans la diégèse, à la façon qu'avait Beethoven de faire entrer, sur la pointe des pieds, son auditeur dans le monde pastoral de sa sixième symphonie grâce à son premier thème. Cette introduction beethovenienne anticipe une citation que nous retrouverons un peu plus tard dans l'oeuvre d'Enyedi (la septième symphonie). Mais c'est aussi, et avant tout, Simon le Hongrois qui arrive à Paris; c'est la rencontre entre deux mondes, entre l'Europe de l'Est et le monde occidental, deux mondes qu'il nous faut regarder « comme si c'était la dernière fois, juste avant [leur] disparition complète et l'émergence de quelque chose de complètement nouveau » (Festival, 71), selon les propos de la réalisatrice. Nous retrouvons ici les considérations économiques relatives au post-communisme énoncées en première partie, transposées dans les territoires de l'esthétique.

Enyedi nous fait partager son amour de Paris dans d'autres séquences. La ville lui sert aussi à montrer le temps qui passe, lorsqu'elle fait se succéder trois vues de Paris prises d'endroits différents, à la lumière de l'après-midi, avec le soleil mourant, et de nuit. Il y a dans ce film, et tout particulièrement dans la façon dont est montrée la ville de Paris, une attention aux détails et une retenue louables. Elle nous fait visiter la ville à travers l'action des personnages, nous promenant des luxueux hôtels particuliers du Parc Monceau aux petits troquets parisiens, dans lesquels Simon s'arrête pour un café ou boire du vin sur des tables recouvertes de toile cirée, entouré de travailleurs jouant aux dés à la lumière d'une ampoule nue.

La vision qu'offre la réalisatrice de la capitale, est fantasmée, puisqu'elle est magique : «le talent de Tibor Máthé, le directeur de la photo, illumine les visions urbaines d'Ildikó Enyedi. Les panoramiques superbes, les plongées vertigineuses, les profondeurs de champ révélatrices, les éclairages rasants sur les façades, les miroitements révélateurs de la transparence des cabines téléphoniques, cet art d'enchanter la matière sert l'univers d'apparences où ressuscite Simon » (Aude 46). Ce n'est pas le Paris de 1998, date à laquelle se déroule l'histoire, mais plutôt le Paris «du Brassaï des années vingt ou de Kertész », comme le remarque justement Stéphane Bouquet (« Simon», 77).

La référence aux années vingt, l'auteure de $A z$ én $X X$. századom ${ }^{11}$ l'assume à nouveau volontiers, et notamment lors d'une séquence burlesque aux accents linderiens, où des éboueurs trouvent Simon, endormi dehors dans une ruelle. L'image subit une très légère accélération, le piano prend plaisir à imiter la musique du cinéma des premiers temps, et les éboueurs lui mettent un carton sous la tête en guise d'oreiller, en prenant garde à ne pas le réveiller. Ici encore, la magie revient puisqu'ils sont mis en scène comme de petits lutins, 
œuvrant dans le silence de la nuit comme dans le conte de Grimm.

Fantasmer Paris, c'est aussi le styliser à l'extrême. Ainsi, Máthé Tibor filme les rues sous des éclairages très soulignés, avec un montage alterné entre les marches de Simon dans les beaux quartiers sous des tons bleutés, et celles de Jeanne dans des quartiers plus populaires et sous des tons ocres : les deux visages de la ville reflètent les deux personnages principaux, et tous les quatre se rencontrent. C'est le cas lorsque Simon sort de la gare, au début du film, et qu'il voit Julie disparaître dans un plan dont la composition rappelle celle de certains plans du Tati de Mon Oncle (1958), qui opposait la ville moderne au village traditionnel français dans un même cadre. Ici, c'est le réalisme de la Gare de l'Est qui se fond dans la magie de Paris : un escalier droit au premier plan où se tient Simon, avec un mur crasseux, tagué, et au second plan - où disparaît Julie - un très beau double escalier en spirale qui monte vers des bâtiments de style hausmannien.

Car la magie de l'amour qui, nous l'avons vu, ne pouvait prendre place qu'à Paris, est en premier lieu celle de la relation entre Simon et Jeanne, et du romantisme à la française qu'elle dégage. Tout dans le prénom - français s'il en est - de «Jeanne » évoque le personnage campé par Julie Delarme. Nous trouvons ici une figure féminine très française, dans cette jolie brune aux yeux bleus, à la fois timide et franche, dont Brigitte Baudin disait qu' «elle a le charme discret et le regard clair des filles du Nord. Elle a l'allure gracile d'une adolescente mais la voix assurée et posée d'une femme qui prend son destin en main. Elle a les pieds sur la terre et la tête dans les étoiles » (22).

Sa relation avec Simon est marquée par le fantasme du Grand Amour, toujours contrecarré, mais toujours victorieux. Dès le départ, «Simon et Jeanne se reconnaissent instantanément. Ils ont la même quête d'amour et d'absolu. J'ai été séduite par la beauté de ces personnages » (22), explique Delarme. Enyedi inscrit leur rencontre dans les codes de la chevalerie et de l'amour courtois, puisque Jeanne est présentée comme une demoiselle en détresse, agressée verbalement par un agent de sécurité, lorsque Simon sort du train. Passant devant sa traductrice sans la voir, il s'approche et, à l'aide d'une magie éthologique, modifie l'attitude de l'agresseur qui passe du hurlement à une galanterie qui prend la forme d'un baisemain. Durant l'agression, Jeanne est filmée en plans serrés qui ne laissent pas voir l'agent, mais qui recentrent l'attention du spectateur sur son regard. L'insertion de contrechamps sur Simon permet à la réalisatrice de faire se croiser leurs regards, suggérant qu'il s'opère déjà entre eux un coup de foudre. Sa disparition dans le plan suivant mêle au caractère soudain de la rencontre l'effacement du geste galant : il la secourt sans qu'elle le sache, elle part sans un mot. Seul un regard les a liés. 
Les modalités de communication entre Simon et Jeanne, tout au long du film, renforcent simultanément la force de leur amour et son apparente impossibilité, impossibilité qui est aussi donnée par la différence d'âge entre les deux personnages ${ }^{12}$. Simon ne parle que le hongrois ; il lui est donc très difficile d'entretenir une conversation avec Jeanne, ce qui est mis en scène avec un plaisir manifeste dans la séquence où Jeanne lui propose son sondage et où il répond au hasard «oui » et «non », se contredisant tant et si bien qu'elle finit par lui demander s'il se moque de lui, situation à laquelle il échappe par une réplique : « café ? ». Dans cette séquence, l'impossible communication qui caractérise le héros, souligne sa solitude dans ce pays qui lui est étranger, et renforce le romantisme de sa rencontre avec Jeanne : le hasard, la chance voire le destin lui font rencontrer une Française dont il tombe amoureux. Mais parler à Jeanne s'avère plus ardu que tous les tours de magie du monde ; c'est ce que nous comprenons lorsqu'il demande à Péter de lui enseigner le français à la place d'un quelconque sortilège, jusqu'à ce que le hasard ou, à nouveau, le destin, lui fasse donner la bonne réponse à la question un peu effrontée de Jeanne : «vous voulez coucher avec moi ? »

Nous arrivons, ou plutôt revenons, au duel final entre Simon et son vieil ennemi, Péter. C'est en tout cas le prétexte donné le plus évidemment à cette séquence finale, mais l'enjeu en est tout autre. Le combat n'est pas tant entre les deux Mages qu'entre les deux magies, entre l'amour et la magie, entre l'homme et la machine, entre la pureté des sentiments et la facticité des vanités que sont ces marchandises de la société du spectacle, la gloire et l'argent.

Pour les besoins de ce duel idéologique, c'est la septième symphonie de Beethoven qui est exhumée. En continuant à se placer dans notre perspective d'une double identification musicale avec les deux magies, l'utilisation d'une œuvre de Beethoven nous apparaît comme un symbole fort de l'essence de la musique instrumentale classique ${ }^{13}$. La dimension dramatique qu'elle installe nourrit naturellement la tension existante à ce moment de l'histoire, mais métaphoriquement elle symbolise la force de l'amour des deux principaux protagonistes, car ce n'est pas la magie de Simon qui le sort de terre. C'est son amour pour Jeanne, qui lui a donné rendez-vous dans trois jours. Il s'agit ici de tuer le mage pour que l'homme puisse vivre, ce qui est judicieusement symbolisé dans l'onirique avant-dernier plan, où Simon marche dans une rue de Paris, et l'on voit Jeanne qui court vers lui, mais lui passe à travers, le faisant disparaître, puis disparaissant à son tour.

Le film se clôt sur une cadence évitée. Jeanne attend Simon à l'endroit convenu; elle cherche du regard, anxieusement et, fixant soudain un point invisible au spectateur, sourit : 
Simon est sorti. Enyedi choisit, pour préserver l'atmosphère d'onirisme et de fantasme du film, de ne pas montrer le baiser final, suivi du traditionnel «fin », mais le spectateur ne s’y trompe pas, la cadence est bel et bien là.

\section{Conclusion}

Dans ce film, la magie est réaliste et l'amour est magique. Nous pourrions dire en guise de conclusion qu'il n'y a pas de message politique ici. Malgré tout, dans les choix qu'opère la réalisatrice, dans les associations d'idées qu'elle met en scène, dans son attention au détail qui fait tout l'intérêt de l'œuvre, il est très tentant de sentir ce film comme le produit de son époque. L'œuvre d'une cinéaste qui, dans un pays commençant à peine à sortir de l'ornière de deux décevantes promesses politiques consécutives (celles du communisme et du capitalisme), s'use à produire plutôt que créer. La critique du matérialisme, de l'attrait de l'argent et plus généralement du système économique occidental est palpable à chaque instant. Mais on sent aussi l'affection qu'a Enyedi pour la solution qu'elle a trouvée : l'envolée dans l'imaginaire, le fantasme, l'amour, Paris, la France.

\section{Bibliographie}

Aude, Françoise. « Simon le mage ». Positif 475 (septembre 2000) : 46-47.

Baudin, Brigitte. «Les charmes subtils de Julie Delarme ». Le Figaro (02/08/2000) : 22.

Boulez, Pierre. «Stravinsky demeure ». Relevés d'apprentis. Paris : Seuil, 1966. 75-183.

Bouquet, Stéphane. «Béla Tarr et sinon ? \ahiers du cinéma 513 (mai 1997) : 57-59.

---. « Simon le mage ». Cahiers du cinéma 548 (juillet-août 2000) : 77-78.

Codelli, Lorenzo. «Mon XX ${ }^{\mathrm{e}}$ siècle ». Positif 341-342 (juillet-août 1989) : 80-81.

---. « Notes festivalières : Budapest 1999 ». Positif 464 (octobre 1999) : 198-199.

Cunningham, John. Hungarian Cinema : From Coffee House to Multiplex. Londres / New York : Wallflower P, 2004. 258.

Dekiss, Jean-Paul. «Le Cinéma hongrois vu par un producteur français en Hongrie en 1996 ». Cahiers d'études hongroises 9 (1997-1998) : 59-62.

Festival internationale del film, Locarno, 52 (1999) : 71.

Horton, Andrew J. «Passive and Pubescent : Peculiar Gender Politics in Central European Cinema ». Kinoeye (17 mai 1999). http://www.cereview.org/kinoeye/kinoeye26old2.html, consulté le 08/07/2015.

Jeancolas, Jean-Pierre. L'œil hongrois : quatre décennies de cinéma à Budapest (1963-2000). Budapest : Magyar Filmunió, 2001. 324. 
Lengyel, David. «De deux sons de cloche : la métaphore dans le cinéma de Béla Tarr». Métaphores et Cultures : en mots et en images. Dir. Véronique Anger et alii. Paris : Harmattan, 2012. 265-84.

Pelinq, Maurice. « Mon XXe siècle ». Jeune Cinéma 179 (juillet 1989) : 29-30.

\footnotetext{
NOTES

${ }^{1}$ Másik János, le compositeur de la musique de Simon Mágus, parle de «pot d'échappement » : la nécessité pour le régime Kádár de relâcher la pression se traduit notamment par un plus grand laxisme vis-à-vis de la censure dans la création cinématographique. (Conversation privée avec Másik János, Forgách András et Kiss Anikó, 23/07/2015.)

2 Fondation pour le cinéma hongrois.

3 Forgách András, lors d'une conversation privée avec Másik János, Forgách András et Kiss Anikó, 23/07/2015. 4 Nous pourrions citer Szász János, Szabó Ildikó, Janisch Attila et bien d'autres.

5 Notons que ce sont en priorité les cinéastes hongrois ayant eu une ouverture sur l'étranger qui ont pu continuer à faire leurs films : Jancsó Miklós, Szabó István et Mészarós Márta principalement.

6 Forgách András, lors d'une conversation privée avec Másik János, Forgách András et Kiss Anikó, 23/07/2015. 7 On se rappelle alors la querelle qui lia (anachroniquement, certes) Proust à Sainte-Beuve autour de la question de la critique de l'œuvre. Je me place ici du côté proustien.

8 L'équivalent hongrois de notre «Pierre».

9 Másik János, lors d'une conversation privée avec Másik János, Forgách András et Kiss Anikó, 23/07/2015.

10 L'utilisation qu'en fait Gustav Malher tendrait à nous faire dire l'inverse, mais généralement les cuivres restent, en instrumentation et dans la famille des vents, beaucoup plus doux que les bois.

11 Où l'on trouvait déjà des références à Griffith, à La petite fille aux allumettes de Jean Renoir (1928), et « un noir et blanc savamment contrasté, nimbe lumineux très hollywoodien pour Dora, éclairage plus plat pour Lili, transitions fortement marquées entre les séquences par la fermeture à l'iris, souvenir discret de quelques film connus » (Pelinq 29).

12 Andrew J. Horton remarque que les relations amoureuses non standard sont un thème souvent abordé dans les films d'Europe centrale des années 90 (Horton).

13 L'appartenance des dernières symphonies de Beethoven à la musique classique ou préromantique a été longuement discutée. Outre le fait que ce n'est pas le propos de ce texte, nous prendrons ici la terminologie de «musique classique » dans son sens le plus vaste, englobant toute la musique savante occidentale depuis la Renaissance jusqu'à la musique contemporaine.
} 